\title{
Simple Sentence Reading and Specific Cognitive Functions in College Students with Dyslexia: An Eye-tracking Study
}

\author{
Sunjung Kim¹ , Linda J. Lombardino² \\ ${ }^{1}$ Department of Communication Sciences and Disorders, University of Central Arkansas, Conway, United States, ${ }^{2}$ University of Florida, Gainesville, \\ United States
}

\begin{abstract}
Slow reading speed of young adults with dyslexia is well-documented. However, most studies have examined comprehension in context of complex text rather than in the context of simple, unambiguous sentences leaving the issue of basic level reading comprehension largely unaddressed. This study was designed to explore the potential contributions of eye gaze, processing speed, and verbal memory to the comprehension of simple sentence reading in college students with dyslexia. Sixty-four college students with and without dyslexia read simple sentences and answered comprehension questions as their eye movements were recorded and they completed tasks of processing speed and verbal memory capacity. Students with dyslexia exhibited longer fixation durations on keywords and showed less efficient scanning patterns between text and questions than their peers. Further, the students' eye patterns were associated with their performance on the cognitive abilities even after controlling for intelligence, verbal ability, and reading abilities. In spite of the simplicity of the experimental reading task, college students with dyslexia showed less efficient eye-gaze patterns and comprehension strategies. Longer eye gaze fixations on keywords in print along with slower general processing speed appears to account for the difference in simple text reading between the two groups.
\end{abstract}

Keywords: Dyslexia, College students, Eye tracking, Processing speed, Verbal memory capacity

\section{INTRODUCTION}

Over $80 \%$ of students with learning disabilities experience difficulty reading [1] and large numbers of these students wish to pursue postsecondary education in spite of their academic challenges [2]. A greater number of students with learning disabilities are enrolling in colleges and universities than ever before [3,4]. Hence, there is a need for more systematic and in-depth study of profiles of students with a specific learning disorder in reading that takes into account cognitive factors that may impact their reading, a core skill for most majors in institutions of higher learning $[5,6]$. In response to this need, we designed a study to investigate reading comprehension skills and cognitive abilities known to play a role in reading in a cohort of college students with dyslexia. We were particularly interested in determining if eye tracking data would provide new insights into well-established slower reading skills in advanced students with dys-

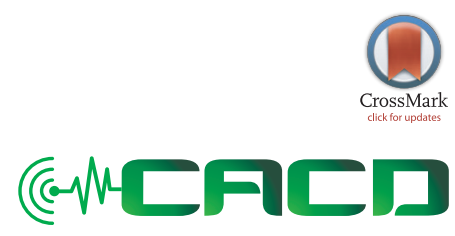

Received: October 18, 2016 Revision: November 29, 2016 Accepted: November 29, 2016

\section{Correspondence:}

Sunjung Kim

Department of Communication Sciences and Disorders, University of Central Arkansas, Box 4985201 Donaghey Ave., Conway, USA

Tel: +5018520850

Fax: +5018520850

E-mail: skim@uca.edu

(C) 2016 The Korean Association of SpeechLanguage Pathologists

This is an Open Access article distributed under the terms of the Creative Commons Attribution Non-Commercial License (http:// creativecommons.org/licenses/by-nc/4.0/) which permits unrestricted non-commercial use, distribution, and reproduction in any medium, provided the original work is properly cited. 
lexia $[7,8]$.

Less efficient reading is manifested by readers' longer eye fixation times on individual words and more frequent eye movements across words, indicators of more effortful cognitive processing [9]. Because eye fixations and movements are believed to reflect underlying cognitive process (10 for review), eye-tracking methodology has been widely used to gather information about readers' micro-level of processing as in word recognitions and phonological parsing as in De Luca, Borrelli, Judica, Spinelli, and Zoccolotti [11] and macrolevel processing as in text comprehension as in Hyönä, Lorch, and Kaakinen [12]. Researchers have reported more and longer eye fixations and higher frequencies of eye movement regressions in students with dyslexia when compared to typical readers $[13,14]$. These studies have provided evidence for a relationship between the complexity of phonological, syntactic, or semantic components of language in text and reading speed in students with dyslexia. However, a more fundamental question regarding reading comprehension in the context of simple sentence forms and content has received far less attention.

By using simple sentences in the current study, we intended to exclude complexity of forms and content as variables and rather to focus on reading efficiency which often remains a challenge, even for the most well-compensated adults with dyslexia [7]. Further, we were interested in the potential contributions of specific cognitive processes to eye gaze fixations on a simple task of reading comprehension. While it is wellknown that inefficient word recognition leads to poor sentence reading [15-17], research in reading comprehension also supports the contributions of non-reading cognitive abilities to individual differences in text reading because the limited cognitive capacities of humans constrain information processing (18 for recent review).

Leonard et al. [19] suggest that cognitive processing abilities have two primary roles, processing speed and processing capacity. Processing speed, the ability to efficiently identify items, make decisions, and move on to the next items, taps a wide range of cognitive behaviors including reading [20]. Catts, Gillispie, Leonard, Kail, and Miller [21] found that poor readers were slower than typical readers on various processing speed measures both on lexical tasks for processing of word naming and meaning and on visual-motor tasks for processing of non-linguistic stimuli. The authors reported that speed of processing accounted for significance variance in reading achievement after controlling for phonological pro- cessing skills.

One of the most widely used tasks for measuring processing speed is the digit-symbol substitution [22]. Studies have shown the task to be minimally affected by intelligence, memory, or attention [23,24]. Digit-symbol substitution has been used extensively to investigate the relationships between general processing speed and aging [ 25 for review] and is often used to investigate the associations between general processing speed and processing of language [26-28]. In their study of relationships between sentence comprehension, verbal working memory capacity, and processing speed using the digitsymbol substitution in college students and older adults, Caplan and Waters [29] reported that verbal working memory was related to syntactic processing and processing speed was related to the overall reading efficiency for both age groups. Processing speed was related to reading efficiency on both an experimental sentence processing task and the standardized test of reading [30, Nelson-Denny Reading Test].

Processing capacity in working memory, the ability to temporarily retain information for mental manipulation [19], is also considered as a significant determinant of higher-order cognition including reading. In their synthesis of research comparing students with and without dyslexia on measures of verbal memory, Swanson, Zheng, and Jerman [31] reported that students with dyslexia were distinctly disadvantaged compared with their peers on the verbal memory and recommended memory be included in the reading assessment protocol. Verbal memory span appears to be a particularly important skill for achieving reading proficiency because readers must be able to retain and manipulate words while accessing their meanings in order to accurately interpret text. Several studies investigating the role of verbal memory in reading have reported that students with dyslexia often have difficulties on tasks of verbal retention [32,33].

Tasks that involve the serial recall of verbal stimuli are among the most expensively used measures for assessing verbal memory capacity in clinical practices and memory research [34]. This type of memory, exemplified by many daily tasks, such as memorizing phone numbers, remembering the sequence of sentences spoken in conversation, and recalling the items on a shopping list, is essential for activities that involve language. The serial recall can be tested in two ways, forward recall and backward recall. The two tasks are often combined into a single score; however, researchers have recently viewed the two tasks separately because the tasks tap different skills. The forward recall primarily involves mainte- 
nance of information while the backward recall is considerably more demanding and involves manipulation as well as maintenance of information, so researchers found performance on backward recall is a more sensitive index of working memory capacity [35] and memory dysfunction [36].

In the current study, we sought to explore relationships between cognitive (processing abilities), behavioral (reading skills), and physiological (eye-tracking) measures to elucidate models of how people with dyslexia comprehend sentences. Previous studies of sentence comprehension have primarily examined how semantic and syntactic complexity would affect reading comprehension of students with dyslexia [37,38]. In contrast, this study was designed to eliminate possible confounding factors for data interpretation due to the complexity of language processing required for tasks by using a linguistically simple set of printed stimuli. Furthermore, previous studies have examined the relationship between reading abilities and cognitive skills by mainly using post-processing measurement (e.g., reading accuracy) [39]. This is a valuable measure of reading performance when using linguistically complex text; however, it may not provide fine-grained levels of information when using simple text because it may not be sensitive enough to detect the reading group differences. By analyzing participants' eye fixations, we attempted to connect individual differences in cognitive abilities to on-line processing of eye gaze patterns of students with and without dyslexia.

In our attempt to further understand how young adults with dyslexia comprehend simple sentences, we designed the current study to employ both behavioral and psycho-physical methods to explore variables that may diminish the abilities of these students to read. The goal of our approach was two-fold: (a) to compare the trajectories of eye patterns in college students with and without dyslexia while they are reading simple sentences and (b) to explore the potential contributions of specific cognitive processing skills, previously reported to be associated with reading ability, to eye fixation patterns during reading the simple sentences for comprehension.

Based on the previous studies $[14,40,41]$, we expected that eye fixation and movement patterns in dyslexia would reflect the students' reading patterns and strategies. Specifically, we expected that the students with dyslexia would be less discriminating than their peers in their eye-gaze fixation times for task-relevant keywords and less-relevant words and that students with dyslexia would show less efficient visual scanning patterns between questions and text when compared to their peers. We also expected that processing speed and memory capacity would be associated to students' eye fixation patterns in sentence reading even after controlling for students' intelligence, verbal ability, and reading skills.

\section{METHODS}

To compare the groups' text comprehension patterns and strategies, we measured each subject's (a) eye gaze at two levels; the sentence level (entire text, text prior to viewing the accompanying question, text after viewing the accompanying question) and the word level (task-relevant keywords and less-relevant words) and (b) visual scanning trajectory including the location of initial fixation and length of the scanpath (i.e., total distance traveled by the eyes while reading sentences). To explore the potential contributions of processing speed and memory capacity to eye gaze patterns, we examined the relationships between the two respective cognitive processes and eye fixation times in three contexts: (a) reading the entire text, (b) fixations on keywords (i.e., areas of interest; AOIs), and (c) fixations on less-relevant words (i.e., non-AOIs) while controlling for intelligence, verbal skill, and reading ability.

\section{Participants}

Sixty-four college students, 31 students with dyslexia (DR) and 33 students with typical reading skill (TR), participated either for course credit or monetary compensation. Of the 31 students with DR, one was excluded from further analyses because he is a non-native speaker of English. Of the 33 students with TR, three were excluded, two due to technical problems with the eye tracking equipment and one due to failure to complete the entire experimental tasks.

The final cohorts of participants with DR and TR were composed of 30 students ( 20 females and 10 males) and $30 \mathrm{stu}-$ dents (23 females and 7 males), respectively. The two groups did not differ in age (mean age of DR group $=22.17$ years, $S D=7.11$, mean age of TR group $=22.10$ years, $S D=2.39 ; \mathrm{F}(1$, $58)=0.50, p=0.48$ ). Students with DR were recruited through emails distributed through the university's disability service office, announcements in undergraduate classes, and posters on campus. Students in the TR group were recruited through a university research participation website. This study was approved by the University's institutional review board.

The first author, a certified speech-language pathologist, along with two trained research assistants, individually tested all potential participants to ensure that they met the inclu- 
sionary criteria for this study. To be included, students were required to meet the following criteria: (1) report a history of reading difficulties beginning in childhood, (2) score at or below one standard deviation of the mean on word reading efficiency or phonemic decoding efficiency tests from the Test of Word Reading Efficiency (42, TOWRE), (3) score no lower than a standard score of 80 on the Brief Intelligence Ability from the Woodcock Johnson III Test of Cognitive Abilities (43, WJ-III-COG), and (4) score no lower than a standard score of 80 on the Verbal Ability from the WJ-III-COG [43].

Students in the TR group reported an absence of a history of reading difficulties at any time while in school. Their status was confirmed by their average or above average scores on the tests administered to the experimental students. When comparing the reading scores of the two groups, students with DR exhibited significantly weaker reading skills as shown in total word reading, $\mathrm{F}(1,58)=150.68, p<0.001$ (DR group $M=80.07, S D=8.44$; TR group $M=102.37, S D=8.76$ ) from TOWRE (42). No difference was found between the groups for the brief intelligence ability score, $\mathrm{F}(1,58)=1.56, p=0.22$ (DR group $M=100.83, S D=8.46$; TR group $M=103.77$, $S D=9.67$ ) or for the verbal ability score, $\mathrm{F}(1,58)=0.45, p=0.51$ (DR group $M=98.10, S D=9.17$; TR group $M=99.70, S D=9.39$ ) from WJ-III-COG (43). All participants in this study were native speakers of English and reported negative histories for pervasive cognitive deficits, behavioral disturbance, neurological illness, psychiatric illness, hearing impairment, or uncorrected visual impairments.

\section{Assessments}

All participants completed three experimental tasks: a sentence comprehension task during which eye tracking data were collected, a processing speed task, and a memory capacity task. A description of these measures follows.

\section{Experimental sentence comprehension task}

This task consisted of 12 stimulus sets (refer to Figure 1). Each set included two statements (e.g., "The girl has six birds, four dogs, and seven turtles. The boy has three birds, five dogs, and one turtle.") accompanied by a comprehension question (e.g., How many dogs does the boy have?). Each sentence contained one subject (boy or girl) and three common objects

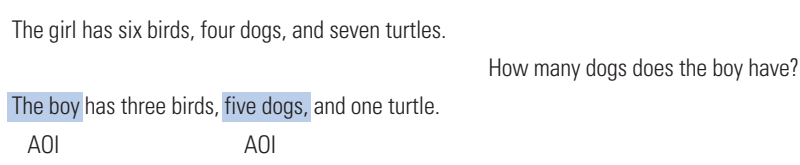

Figure 1. Sample experimental reading task and areas of interest (AOIs) designated for eye tracking analysis. AOls are the word areas in the sentences needed to answer the accompanying question. The rest of the words were designated as non-AOls. The accompanying question was not included in the AOls or Non-AOls.

(e.g., dogs, turtles, birds). Both sentences in a set were composed of ten-word utterances and were positioned on the left side of the computer screen in a double-spaced format and the accompanying question was positioned on the right side of the screen. To minimize memory constraints, the sentences were presented as a whole, instead of word-by-word presentation as it corresponds more closely to the natural reading situations and the question was included on the same screen as the set of statements, rather than appearing on a separate screen. The mean frequency count (per million) of the words in the statements and questions is 105.66 (44, Celex).

The experiment items were combined twelve filler items. The filler item consisted of a single sentence (e.g., "The boy ate one apple, two cherries, and three bananas.") and a question (e.g., "Did the boy eat more cherries than bananas?") to prevent participants from discerning the fact that all of the experimental statements have the same schematic firm. Experimental items and filler items were counterbalanced using a Latin square to control for the variability. All items were presented in a pseudo-randomized order. To avoid unintended effects of item order, sentence sets were divided into sets A and $B$ in which items were listed in two different orders. Half of the participants in each respective groups were tested with set $\mathrm{A}$ and the other half were given set $\mathrm{B}$ (DR group $n$ in set $\mathrm{A}=14$ and $n$ in set $\mathrm{B}=16$; TR group $n$ in set $\mathrm{A}=17$ and $n$ in set $B=13)^{1)}$. For each of the comprehension questions, each participant's response to the comprehension question received a score of either 1 (correct answer) or 0 (incorrect answer). The maximum possible score was 12 across all trials.

\section{Processing speed}

Digit-symbol substitution [22] is a timed test used to measure

\footnotetext{
${ }^{1)}$ To confirm that there was no significant difference between sets A and B data, we compared participant data for the sets in each group. For the DR group, no difference was found between sets in age, $\mathrm{F}(1,28)=2.75, p=0.11$, word reading efficiency, $\mathrm{F}(1,28)=3.18, p=0.09$, or experimental sentence reading accuracy, $\mathrm{F}(1,28)=0.71$, $p=0.41$. For the TR group, no difference was found between sets in age, $\mathrm{F}(1,28)=1.31, p=0.26$, word reading efficiency, $\mathrm{F}(1,28)=0.44, p=0.51$, or experimental sentence reading accuracy, $\mathrm{F}(1,28)=0.06, p=0.82$.
} 
the participants' processing speed. For the digit-symbol substitution, the participant was presented with a sheet of paper showing nine numbers paired with symbols on the top of the page and told to copy the corresponding symbols in boxes adjoining to the numbers shown below. The total score was the number of correct number-symbol matches in 90 seconds. Test-retest reliability for digit-symbol substitution was reported to range from .82 to $.88[45,46]$.

\section{Memory capacity}

The digit span backward subtest from the Wechsler Memory Scale [47] was used to measure the participants' processing capacity. The experimenter read a series of increasingly long lists of numbers at a speed of one second per digit. The participant was then asked to repeat the numbers in the reverse order (two trials for item, 2 to 8 digits). The test was terminated when the participant failed to replicate sequences containing the same number of digits twice. The total score was the number of lists repeated correctly, with a maximum score of 14 . Test-retest reliability was reported at $.83[48,49]$.

\section{Eye-tracking apparatus}

Eye movements were measured with an LC Technologies head-free EyeFollower binocular system operating at $120 \mathrm{~Hz}$ with a 0.45 degree gaze-point tracking accuracy throughout the operational head range (horizontal range: 30 inches and vertical range: 20 inches). Text was presented in black Arial 20 point font on a 24 inch $(61 \mathrm{~cm})$ light-emitting diode (LED) monitor with a resolution of $1,920 \times 1,080$ pixels. Fixations were extracted with a temporal threshold of $100 \mathrm{~ms}$ and a spatial dispersion threshold of $1.5^{\circ}$ (minimum deviation of 25 screen pixels). NYAN 2.0 software from Interactive Minds Eyetracking Solutions was used to analyze eyegaze data.

\section{Procedure}

All tests were administered individually. Testing was conducted in approximately one hour. Language tests, and a brief measure of intelligent ability, and eye tracking tasks were administered followed by tests of processing speed and memory capacity. Administration procedures for language and intelligence tests followed standardized procedures described in the test manual. Prior to the eye tracking task for sentence comprehension, the experimenters explained the eye-tracking methodology to the participants. Participants sat at a distance of 23.62 inches $(60 \mathrm{~cm})$ from the LCD monitor and used a custom-designed keyboard. After a thirteen-point calibra- tion procedure, a trial practice was conducted on four items to familiarize participants with the procedures. The experimenter provided verbal feedback to the participants on the practice items regarding the accuracy of their answers. For the experimental trials, the experimenter instructed participants to read the text and answer the accompanying question as rapidly and as accurately as possible; however, no time limit was given. To avoid a possible bias related to participants' hand dominance, participants were instructed to press the spacebar with their dominant hand. The participants controlled the timing of the presentation for each stimulus by pressing the spacebar to advance the program after completing a trial.

\section{RESULTS}

Sentence comprehension accuracy scores for DR and TR groups were $11.83(S D=0.38)$ and $11.73(S D=0.52)$, respectively (maximum possible points $=12.0$ ). The group means did not differ, $\mathrm{F}(1,58)=0.7, p>0.05$. In contrast, reaction times significantly differed for the two groups, $\mathrm{F}(1,58)=8.44, p=$ 0.005 . It took an average of $6,764.60 \mathrm{~ms}(S D=1,669.69)$ per trial for the DR group while an average of 5,642.65 ms ( $S D=$ 1,298.49) per trial for the TR group.

\section{Eye fixation analyses on sentence and word levels}

Eye fixation measures were analyzed in two levels: the sentence level and word level, using total gaze duration (50, sum of the durations of fixations in a region) and total number of fixation measures (50, sum of fixation count in a region). These eye gaze measures are considered to be important as global measures of visual attention [51]. For sentence level measures, we calculated total gaze duration and total number of fixations on (a) the entire text, (b) text before participants viewed the accompanying question, and (c) text after participants viewed the accompanying question. For the word level, we calculated total gaze duration and total number of fixations on (a) the Areas of Interest (AOIs) and (b) non-AOIs. AOIs are the keyword areas on the sentence needed to answer the accompanying question and all other areas of the sentence were designated as the non-AOIs. For example, to answer the question "How many dogs does the boy have?," participants needed to look at two areas, the location of the agent (e.g., the boy) and the location of the correct object response (e.g., five dogs) in the text, "The girl has six birds, four dogs, and seven turtles. The boy has three birds, five dogs, and one 

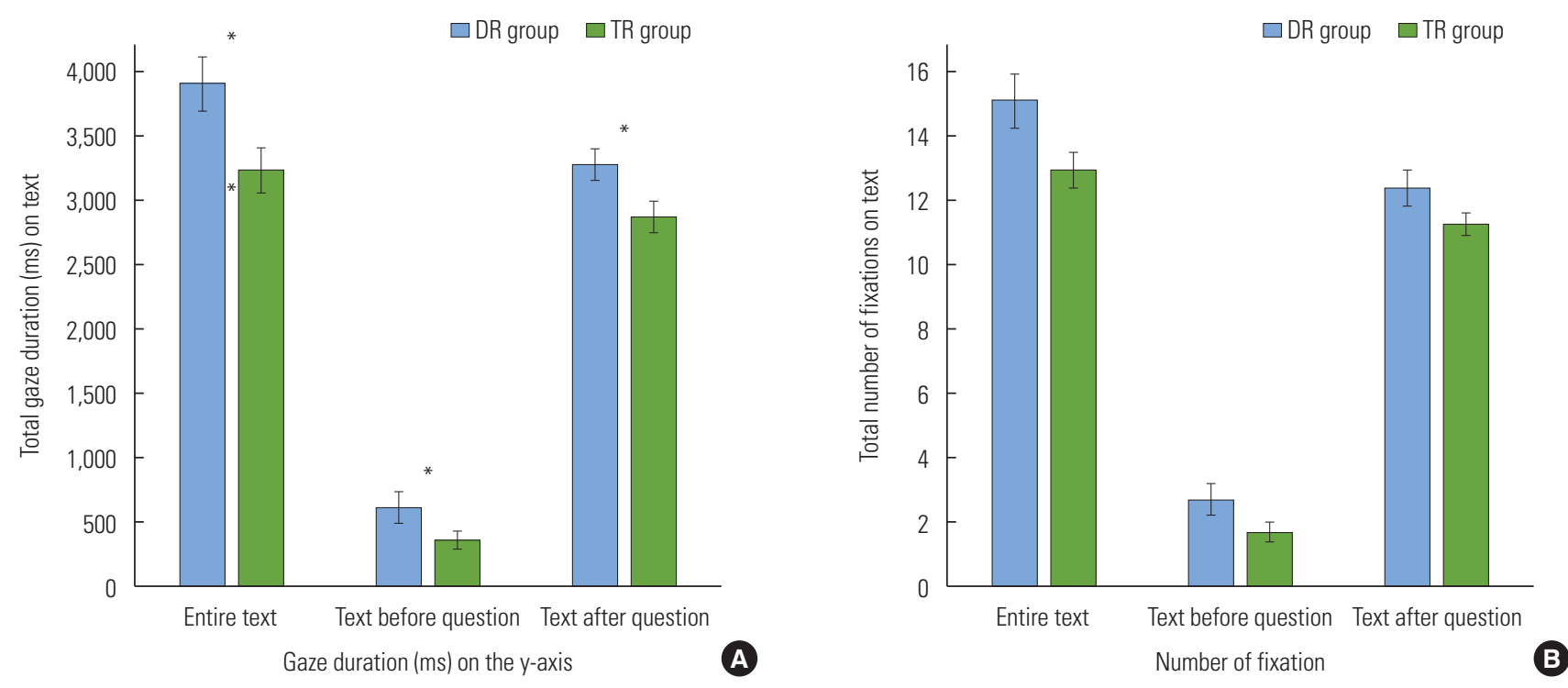

Figure 2. Total gaze duration (in milliseconds; $\mathrm{ms}$ ) and total number of fixations on the entire text, text before viewing the accompanying question, and text after viewing the accompanying question for DR (dyslexia) group and TR (typical reading) group (bars indicate standard errors of the mean). * $p<0.05$.

turtles." Thus, in this example, 'the boy' and 'five dogs' are designated as AOIs and other words as non-AOIs. The examiner (1st author) excluded from analysis any responses that were answered incorrectly. This occurred in $0.9 \%$ of the data. Further, the examiner visually inspected each trial to check that the tracker had correctly recorded eye movements. Approximately $6 \%$ of data was excluded due to track loss or program error.

Total gaze durations and total number of fixations on (a) the entire text, (b) text prior to viewing the accompanying question, and (c) text after viewing the question are presented in Figure 2. The first set of analysis was performed to compare the two groups for duration and number of eye fixations at the sentence level. Analyses of covariance (ANCOVAs) were conducted to compare the DR and TR groups on gaze fixation measures with group membership (DR and TR groups) as the independent variable, eye fixation measures as the dependent variable, and WJ-III-COG verbal ability score (43) as the covariate. The verbal score was included to ensure that our results were not attributable to differences in verbal comprehension ability. The distribution of the processing data is commonly incompatible with a normal distribution [52], thus we converted the eye data to a normal distribution $(-1.0$ $<$ skewness < 1.0), using a logarithmic transformation [53].

The DR group had significantly longer gaze duration times than the TR group in (a) the entire text, $\mathrm{F}(1,57)=7.75, p=0.007$, $\eta_{\mathrm{p}}{ }^{2}=0.12$, (b) text prior to viewing the question, $\mathrm{F}(1,57)=4.35$, $p=0.04, \eta_{\mathrm{p}}{ }^{2}=0.07$, and (c) text after viewing the question, $\mathrm{F}(1$,
57) $=5.61, p=0.02, \eta_{\mathrm{p}}{ }^{2}=0.10$. The covariate, verbal ability, was not significantly related to any of the three eye gaze variables, all ps $>.05$. In contrast, the groups did not differ on the total number of fixations in (a) the entire text, $\mathrm{F}(1,57)=3.87$, $p=0.06$, (b) text prior to viewing question, $\mathrm{F}(1,57)=3.88$, $p=0.06$, or (c) text after viewing the question, $\mathrm{F}(1,57)=2.44$, $p=0.12$. Again, verbal ability was not significantly related to any of the frequency of eye gaze fixations, all $p>0.05$.

The second set of analysis was performed to examine locus of eye-gaze fixations at the word level. Eye gaze for words was measured in two areas, areas of interest (AOIs) and non-AOIs. AOIs included the two keywords to answer the accompanying question, the location of the agent (e.g., the boy) and the location of the correct object response (e.g., five dogs) and nonAOIs included the other words in the sentences (refer to Figure 1). The measures were computed as a total gaze duration per word to provide adjustment for differences in the number of words across AOIs and non-AOIs. Total gaze durations on the AOIs and non-AOIs are presented in Figure 3.

The DR group had significantly longer gaze duration times than the TR group on the AOIs and non-AOIs, $\mathrm{F}(1,57)=5.05$, $p=0.02, \eta_{\mathrm{p}}{ }^{2}=0.08 ; \mathrm{F}(1,57)=7.18, p=0.01, \eta_{\mathrm{p}}{ }^{2}=0.11$, respectively. Verbal ability was not significantly related to the total gaze duration on the AOIs or non-AOIs, all ps $>.05$. For total number of fixation on the AOIs, the two groups were not different, $\mathrm{F}(1,57)=3.07, p=0.09$; however, on the non-AOIs, the DR group had significantly more numbers of fixation than the TR group, $\mathrm{F}(1,57)=4.72, p=0.03, \eta_{\mathrm{p}}{ }^{2}=0.08$. Again, verbal ability 

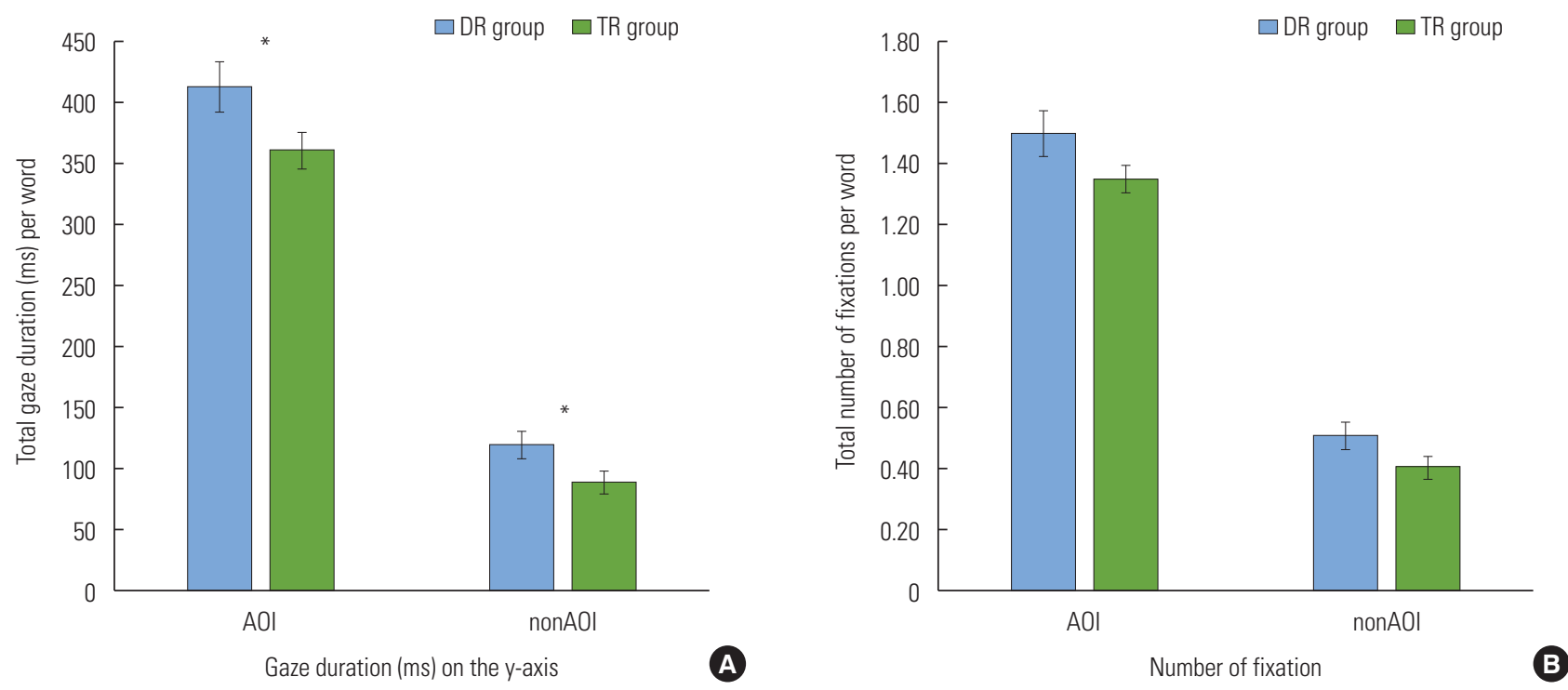

Figure 3. Total gaze duration (in milliseconds; $\mathrm{ms}$ ) and total number of fixations for the areas of interest (AOls) and non-AOls for DR (dyslexia) group and TR (typical reading) group (bars indicate standard errors of the mean). * $p<0.05$.

was not significantly related to the frequency of eye gaze fixations for the AOIs or non-AOIs, all ps $>0.05$.

In summary, analyses of the eye-fixation patterns of the DR and TR groups showed that, in spite of the ease of the task, college students with DR were slower to read sentences in responding to a simple comprehension question. Specifically, they fixated for longer period of time on the text than their peers both before and after reading questions; however, they did not move their eyes more frequently between words. Hence, the slower speed of the DR was due to the time needed to identify word (i.e., immediate word recognition), rather than the need for multiple fixations on a word. Second, students with DR spent longer times fixating on both task-relevant keywords (i.e., AOIs) and less-relevant words (i.e., nonAOIs) than their peers.

\section{Initial fixation location and scanpath length analyses}

Location of the first fixation was analyzed to determine participants' initial searching strategy for this task. We manually analyzed participants' initial fixation points on 720 (60 participants $\times 12$ stimuli) data points. First fixation points were categorized as fixation on the text area or fixation on the question area. Among 720 data, 18 were excluded because their initial viewing points were neither in text nor question areas. The remaining 702 data were used for analysis (354 for DR group and 348 for TR group). For the DR group, the first fixations were located in text for 233 trials (66\%) and in question for 121 trials (34\%). For the TR group, the initial first fixations were lo- cated in text for 197 trials (57\%) and in question for 151 trials (43\%). The group membership (DR and TR) $\times$ location (text and question) two-way loglinear analysis yielded a significant main effect for location and a two-way interaction effect for group membership $\times$ location. More students inspected the text region before the question region, $x^{2}(1)=35.86, p<0.01$, while a great number of students from the TR group inspected the question region first in comparison to the DR group, $x^{2}(1)=6.28, p=0.01$.

Scanpath, the summation of the distance between gazepoints, indicate readers' scanning and visual search strategies [54]. Lengthy scanpaths indicate increased sequence of eye movements (i.e., saccades) or poor layout design [55]. The average scanpath lengths (in pixel) for the DR and TR groups were $4783.61(S D=1,139.64)$ and 4,439.78 ( $S D=972.01)$, respectively. ANCOVA with group membership (DR and TR) as the independent variable, scanpath length as the dependent variable, and WJ-III-COG verbal ability scores (43) as the covariate indicated that the scanpath length of the two groups did not differ, $\mathrm{F}(1,57)=1.43, p=0.23$. The covariate, verbal ability, was also not significantly related to the scanpath length, $\mathrm{F}(1,57)=0.20, p=0.66$.

\section{Relationships between sentence eye gaze duration and cognitive processing abilities}

Prior to exploring relationships between processing speed, memory capacity, and sentence comprehension, we compared the DR and TR groups' scores on these two cognitive 
measures. For the digit-symbol substitution task, the average raw score (total possible score $=93$ ) was $62.33(S D=8.29)$ for the DR group and $68.03(S D=10.00)$ for the TR group. On the verbal memory task, the average digit span backward scores (maximum possible score $=14$ ) was $6.80(S D=2.36)$ for the DR group and $7.00(S D=1.59)$ for the TR group. To test for group differences, the raw scores from the digit-symbol substitution and digit span backward tasks were scaled to a mean of 0 and a standard deviation of 1 . ANCOVAs were used with group membership (DR and TR) as the independent variable, cognitive tests as the dependent variable, and WJ-III-COG brief intelligence ability score (43) as the covariate. The brief intelligence ability score was included to ensure that the group difference in the two cognitive abilities was not attributable to differences in general intelligence. We found that the DR group had a significantly lower score on the digit-symbol substitution than the TR group, $\mathrm{F}(1,57)=4.14, p=0.04, \eta_{\mathrm{p}}{ }^{2}=0.07$ and the covariate, brief intelligent ability, was also significantly related to the digit-symbol substitution score, $\mathrm{F}(1$, $57)=11.51, p=0.001, \eta_{\mathrm{p}}{ }^{2}=0.17$. In contrast, the groups did not differ on the digit span backward memory task, $F(1,57)=0.03$, $p=0.87$ and brief intelligence ability was not significantly related to the digit span backward score, $\mathrm{F}(1,57)=1.85, p=0.18$.
Subsequent analyses were conducted to determine the contribution of cognitive processing measures to gaze duration for the entire text, AOIs, and non-AOIs. Multicollinearity and independent errors assumptions were examined prior to the regression analyses. To test for multicollinearity, the variance inflation factor (VIF) was estimated. All values of the VIF were close to 1.0 , suggesting multicollinearity was not biasing the regression model [56]. To test for independent errors, the Durbin-Watson test was used to examine serial correlations between errors. All values of the Durbin-Watson test were between 1 and 3, suggesting the residuals in the model were independent [57]. A series of hierarchical regression analyses were used to investigate the unique contribution of individual variables. The brief intelligence ability score and verbal ability score from WJ-III-COG (43) were entered for step 1, the reading group (DR coded 1 and TR coded 2) was entered for step 2, and digit-symbol substitution and digit span backward scores were added for step 3. Table 1 shows the regression results for the total gaze duration on the entire text, AOIs, and the nonAOIs that served as outcome variables.

For the total gaze duration on the entire text, the step 1 analysis was significant, $R^{2}=0.18, \mathrm{~F}(2,57)=6.40, p=0.003$. The beta weight for the brief intelligence ability was significant, $\beta=-0.60$,

Table 1. Hierarchical multiple regression analyses for intelligence, verbal ability, reading skills, processing speed, and memory capacities predicting total gaze duration for sentence and word levels

\begin{tabular}{|c|c|c|c|c|c|c|}
\hline & \multicolumn{6}{|c|}{ Total gaze duration } \\
\hline & \multicolumn{2}{|c|}{ Entire text (sentence level) } & \multicolumn{2}{|c|}{ AOls (word level) } & \multicolumn{2}{|c|}{ Non-AOIs (word level) } \\
\hline & $\Delta R^{2}$ & $\beta$ & $\Delta R^{2}$ & $\beta$ & $\Delta R^{2}$ & $\beta$ \\
\hline Step 1 & $0.18^{* *}$ & & $0.19^{* *}$ & & $0.14^{*}$ & \\
\hline Brief Intelligence Ability & & $-0.60^{* *}$ & & $-0.60 * *$ & & $-0.51^{* *}$ \\
\hline Verbal Ability & & $0.51^{* *}$ & & $0.55^{* *}$ & & $0.46^{*}$ \\
\hline Step 2 & $0.08^{* *}$ & & $0.05^{* *}$ & & $0.08^{* *}$ & \\
\hline Brief Intelligence Ability & & $-0.54^{* *}$ & & $-0.55^{* *}$ & & $-0.45^{*}$ \\
\hline Verbal Ability & & $0.49^{* *}$ & & $0.54^{* *}$ & & $0.45^{*}$ \\
\hline Reading group & & $-0.29 *$ & & $-0.23^{\dagger}$ & & $-0.29^{*}$ \\
\hline Step 3 & $0.10^{* * *}$ & & $0.07^{* *}$ & & $0.08^{* *}$ & \\
\hline Brief Intelligence Ability & & -0.34 & & $-0.47^{*}$ & & -0.22 \\
\hline Verbal Ability & & 0.32 & & $0.44^{*}$ & & 0.27 \\
\hline Reading group & & $-0.23^{*}$ & & -0.20 & & -0.22 \\
\hline Digit-Symbol substitution & & $-0.30^{*}$ & & -0.15 & & $-0.32^{*}$ \\
\hline Digit span backward & & $0.23^{*}$ & & $0.23^{*}$ & & 0.12 \\
\hline Total $R^{2}$ & $0.38^{* * *}$ & & $0.31^{* *}$ & & $0.30^{* *}$ & \\
\hline
\end{tabular}

$\Delta R^{2} ; \mathrm{R}$ square change; AOls, Areas of Interest.

${ }^{\dagger} p=0.05,{ }^{*} p<0.05,{ }^{* *} p<0.01,{ }^{* * *} p<0.001$. 
$t=-3.52, p=0.001$, indicating that the higher the participants' intelligence ability, the less time they spent fixating on the text. The beta weight for the verbal ability was significant, $\beta=0.51$, $t=2.96, p=0.004$, indicating that the higher the participants' verbal ability, the more time they spent fixating on the text. The step 2 analysis also yielded a significant result, $R^{2}=0.27, \mathrm{~F}(3$, $56)=6.77, p=0.001$. As in the step 1 , the beta weights for the brief intelligence ability and verbal ability were significant, $\beta=-.54, t=-3.28, p=0.002 ; \beta=0.49, t=2.99, p=0.004$, respec tively. The beta weight for the reading group was significant, $\beta=-.29, t=-2.51, p=0.01$, indicating that the DR group spent significantly longer time on the entire text than the TR group. Lastly, the step 3 analysis was significant, $R^{2}=0.38, \mathrm{~F}(5$, $54)=6.49, p<0.001$. The reading group significantly predicted total gaze duration on the entire text, $\beta=-.23, t=-2.04, p=0.04$. The beta weight for the digit-symbol substitution was significant, $\beta=-.30, t=-2.18, p=0.03$, indicating that the higher the digit-symbol substitution score was, the less time participants spent fixating on the text. The beta weight for the digit span backward was significant, $\beta=0.23, t=2.05, p=0.04$, indicating that the higher the digit span backward score was, the more time participants spent fixating on the text. Neither intelligence nor the verbal ability significantly predicted the total gaze duration on the entire text, all ps $>0.05$.

For the total gaze duration on the AOIs, the step 1 analysis was significant, $R^{2}=0.19, \mathrm{~F}(2,57)=6.86, p=0.002$. The beta weights for the brief intelligence ability and verbal ability were significant, $\beta=-0.60, t=-3.55, p=0.001 ; \beta=0.55, \mathrm{t}=3.26$, $p=0.002$, respectively. The step 2 model was also significant, $R^{2}=0.25, \mathrm{~F}(3,56)=6.05, p=0.001$. Brief intelligence ability significantly predicted the total gaze duration on the AOIs, $\beta=-.55$, $t=-3.32, p=0.002$, as did verbal ability, $\beta=0.54, t=3.26$, $\mathrm{p}=0.002$. The reading group was marginally significant, $\beta=-.23$, $t=-1.94, p=0.05$. Lastly, on the step 3 , all predictors were entered simultaneously, resulting in a significant increase in $R^{2}$, $R^{2}=0.31, \mathrm{~F}(5,54)=4.93, \mathrm{p}=0.001$. Intelligence ability, $\beta=-0.47$, $t=-2.39 p=0.02$, verbal ability, $\beta=0.44, t=2.51, p=0.02$, and digit span backward, $\beta=0.23, t=2.03, p=0.04$, were significant predictors. Neither reading group nor digit-symbol substitution significantly predicted the total gaze duration on the AOIs, all ps $>0.05$.

For the total gaze duration on the non-AOIs, the step 1 analysis indicated that two predictors explained $14 \%$ of the variance, $R^{2}=0.14, \mathrm{~F}(2,57)=4.52, p=0.01$. Brief intelligence ability significantly predicted the total gaze duration on the non-AOIs, $\beta=-0.51, t=-2.89, p=0.005$, as did verbal ability, $\beta=0.46$, $t=2.64, p=0.01$. On the step 2 , reading group was added to the predictors, resulting in a significant increase in $R^{2}, R^{2}=0.22, \mathrm{~F}(3$, 56) $=5.21, p=0.003$. Brief intelligence ability, $\beta=-0.45, t=-2.63$, $p=0.01$, verbal ability, $\beta=0.45, \mathrm{t}=2.65, p=0.01$, and reading group, $\beta=-0.29, t=-2.41, p=0.01$ were significant. Lastly, the step 3 analysis was significant, $R^{2}=0.30, \mathrm{~F}(5,54)=4.63, p=0.001$. Digit-symbol substitution was the only significant predictor, $\beta=-0.32, t=-2.23, p=0.03$. Other variables did not predict the total gaze duration on the non-AOIs, all ps $>0.05$.

In summary, the hierarchical regression analyses revealed that the DR group took longer time to process the entire text than the TR group. Processing speed was negatively related to the non-AOIs, indicating that students with faster processing speed skills spent less time fixating on the less-relevant words. Memory capacity was positively related to the AOIs, but not to the non-AOIs, indicating that students with larger processing capacity spent more time engaged in the task-relevant keywords, but not in the less-relevant words.

\section{DISCUSSION}

Reading time is the temporal dimension of comprehension, rather than a separate aspect of reading [58]. A change in one dimension leads to a change in another so that any variations in demands on comprehension should affect reading time, and vice versa. In our attempt to further understand how young adults with dyslexia spend their times on text compared to their peers with typical reading skills, we used behavioral and psycho-physical measures to explore variables that may impact reading times. To this end, we explored answers to specific questions associated with simple sentence reading of postsecondary students. We examined differences in eye gaze times during simple sentence reading in students with and without dyslexia and explored contributions of the students' cognitive processing skills to their eye gaze times on task-relevant and less-relevant information.

As we predicted, college students with dyslexia read less strategically than their peers with typical reading skills. First, regardless of whether the words in sentences were task-relevant keywords or not, students with dyslexia spent significantly longer time on the words than their peers. They did not show strategic reading patterns such as spending more times on keywords or less times on non-keywords. Second, while typical readers often began their task by examining the accompanying comprehension question such that they could find which words of the given text were the informative parts 
to answer the question, fewer students with dyslexia initially used this strategy to facilitate efficient comprehension. Third, the students with dyslexia spent more time reading the text, even before reading questions. That is, they did not quickly shift their eye gaze from text to the questions to locate keywords needed to answer the questions. In contrast to the finding that skilled readers are able to adjust their inspection strategy according to the purpose of reading and the task demands, students with dyslexia were less adept at using this strategy for enhancing reading comprehension efficiency [59].

Our findings support the importance of teaching students metacognitive strategies associated with successful reading and learning. Specifically, it is important that students develop metacognitive monitoring and control skills, including identifying keywords in text to answer comprehension questions accurately and efficiently and looking over questions before reading text to access key points of focus and to determine irrelevant details. Sheffield [60] pointed out that students with dyslexia need explicit and extended instruction and training for reading strategies in order for them to be able to execute the strategies with automaticity.

In addressing metacognitive skills needed for reading, Mokhtari and Reichard [61] described three types of strategies: global reading strategies, problem-solving strategies, and support reading strategies. Global reading strategies set the stage for the act of reading such as deciding what to read closely and what to ignore, problem-solving strategies are localized repair techniques used when difficult arise in understand text (e.g., checking understanding when coming across conflicting information) and support reading strategies require the use of outside reference materials to maximize text comprehension (e.g., using dictionaries or library materials). Mokhtari and Reichard [61] investigated whether the use of the three reading strategies differ depending on the reading level for 825 students. They found that skilled readers used the global and problem-solving strategies more frequently than less skilled readers. Currently, most studies regarding the efficacy of employing such reading strategies used the self-report measures [62]. In future research, direct observational studies should be conducted to investigate the efficacy of teaching metacognitive reading strategies, especially for poor readers given that instructional procedures reported for using these strategies are time-consuming and work-intensive for both educators and students [61,62].

Analysis of eye gaze patterns in the current study showed that students with dyslexia fixated for longer periods of time on the keywords in the sentences essential for responding accurately to the questions. Interestingly, however, the students did not show a larger number of fixations on the keywords than their peers in spite of their prolonged durations. Typically, duration of eye fixations is positively correlated with the number of fixations [9]. Hyönä and Olson [14] observed that correlations between duration and number of eye fixations are particularly strong when the target stimuli are both informative and difficult to process. In the current study, while the keywords were informative for answering the questions, they were highly familiar words that should have been easy to process. It is likely that the simplicity of the lexicon used in the experimental sentences accounted for the lack of group differences for total number of eye fixations in spite of the longer fixation times needed to process keywords observed for the students with dyslexia. These findings suggest that once students with dyslexia recognized keywords, they were able to retain the words' orthographic and/or phonological memories. The scanpath length analyses for gaze patterns support the lack of difference in the number of eye movements between the two groups. Slow recognition of lexically complicated or syntactically ambiguous words in young adults with dyslexia has been reported [e.g., 36,61]. Data from this study support previously cited research showing that college students with dyslexia show delayed word recognition while reading text. Our results showed that (a) slow word recognition occurs even in the context of a very simple sentence reading task and that (b) rate of word recognition alone, rather than both rate of word recognition and the number of refixations on words, accounts for the diminished reading speed in a simple sentence reading of students with dyslexia.

Consistent with previous reports of processing speed deficits in poor readers [e.g.,25,26,62,63], our students with dyslexia were significantly slower than their control peers on the behavioral measures of processing speed. While depressed processing speed on cognitive behavioral measures has also been reported for students with other types of learning disabilities [e.g.,64-66], slow processing speed is more apparent in individuals with dyslexia $[20,69]$. In a comparative study of processing speed ability in students with dyslexia and students with attention deficits/hyperactivity disorder, Shanahan et al. [20] reported that both groups showed deficits in processing speed but students with reading disabilities demonstrated more severe processing speed deficits.

In contrast, no difference was found between the groups' performance on our tasks of verbal memory span. Even 
though several studies have found that verbal memory is related to reading ability [70-72], the relationship is likely to depend on numerous factors including reading components and participants' characteristics [73]. Our data concur with findings from Alloway et al's [74] study in which they reported no differences between college students with and without dyslexia on measures of verbal working memory. A possible explanation for the two groups' similar performance on tests of memory involves dissociation between word reading and text comprehension. In their longitudinal study to examine factors that account for variance in word reading and text comprehension, Oakhill, Cain, \& Bryant [75] found that verbal working memory contributed to text comprehension, but not to word reading. In the current study, the two groups were classified, in large part, by their word reading efficiency skills. Poor word reading should not be expected to predict verbal working memory ability in all populations.

Finally, when we investigated relationship between the two cognitive abilities and eye fixations on sentence reading, consistent with previous studies showing that non-reading cognitive abilities impact reading performance ( 31 for recent review), our regression analyses showed that specific cognitive processing skills accounted for unique variance in predicting eye fixation times. These relationships survived while controlling for intelligence and verbal abilities. Specifically, for eye gaze times on the entire text, reading group, processing speed, and memory capacity were significant predictors. Memory capacity was the significant predictor for task-relevant keywords (i.e., AOIs) and processing speed was the significant predictor for less-relevant words (i.e., non-AOIs). Both processing speed and memory capacity were related to the eye gaze times; however, the dynamic of the relationship was different. The faster the speed of processing, the shorter the time the students spent on the less-relevant words. The larger capacity for verbal memory of digits, the longer the time the students spent on the task-relevant keywords, but not on the lessrelevant words.

Processing speed is a cognitive skill which determines how quickly a person can complete a given task [23]. This speed is crucial for efficient word recognition in text. The task of reading in an alphabet language requires the serial mapping of visual codes (i.e., graphemes) onto a language's sound systems (i.e., phonemes). According to the visual-verbal disconnection hypothesis [76], slower reading in dyslexia is due to a disruption in an internal timing mechanism for coordinating orthographic symbols with the phonological codes. Breznitz and colleagues [77-80] have investigated the timing mechanism extensively in individuals with dyslexia using a visualverbal asynchrony model with event-related potentials measurement. Based on their findings, particularly for the slower P300 latencies, serving as a temporal measure of neural activities underlying the perceptual processing speed, they concluded that depressed processing speed is an underlying factor of dyslexia. So far, there has been little attempt to connect individual differences in cognitive abilities to the patterns of eye gaze [39]. Our psychometric processing speed data in conjunction with our eye fixation data suggest that rapid serial visual-motor processing (behavioral tasks) and rapid serial ocular-motor processing (eye fixation patterns) for recognizing and categorizing simple symbols share features that contribute to depressed reading time.

Verbal memory was positively related to the reading time. That is, students with higher verbal memory span spent more time engaged in task-relevant text information, but not in lessrelevant text information. Verbal memory capacity taps, in part, attention control [81,82, see also, 83]. Greater working memory capacity results from greater ability to maintain taskrelevant information in action while avoiding distraction. According to Engle and Kane [84], two components of attentional control are related to working memory capacity. The first component was goal maintenance in active memory and the second component was the resolution of response conflict. Engle and Kane linked the two components to the brain structures suggesting that the goal maintenance relies on prefrontal cortex circuitry while the competition resolution relies on anterior circulate. In our study, students with higher verbal memory span maintained task-relevant information in active memory longer than students with lower verbal memory span. Using the Engle and Kane's component model, our data appear to reflect that the students' verbal memory capacity is related to their goal maintenance ability. However, further studies are needed to better understanding interrelationships among working memory processes, higher other cognitive abilities such as reading, and the neurological substrates that subserve theses skills.

\section{CONCLUSIONS}

Eye fixation and movement measures taken during sentence reading expanded our understanding of roles that specific cognitive behaviors play for component skills of reading. The primary contributions of this report are the findings that (1) 
college students with dyslexia read simple words in short sentences more slowly than their typical reading peers even though the sentences consisted of elementary words, (2) this difference co-occurs with their slower times on a nonlinguistic test of processing speed, and (3) performance on tests of processing speed and verbal memory span accounts for unique variation in their eye fixations during sentence reading. Students with faster processing speed skill fixated for shorter periods of time on less-relevant text information and students with larger verbal memory spans fixated longer on more-relevant information. Further studies are needed to investigate the influence of cognitive abilities on individual differences in eye gaze patterns during sentence reading across text that varies in complexity, length, and familiarity of content to improve our ability to identify and differentiate among the multiple mechanisms that underlie reading difficulties in adults who are facing the challenges of higher education.

\section{CONFLICT OF INTEREST}

The author has no conflict of interests.

\section{ACKNOWLEDGMENTS}

This study was funded by University of Central Arkansas Research Council, awarded to the first author (URC 348R03). We would like to thank Christina Gomes and Jennie Le for assistance with data collection and participant recruitment. Thanks also to all students who participated in this study.

\section{REFERENCES}

1. Kavale KA, Reese JH. The character of learning disabilities: An Iowa profile. Learn Disabil Q. 1992 May 1;15(2):74-94.

2. Stodden RA, Conway MA, Chang KB. Findings from the study of transition, technology and postsecondary supports for youth with disabilities: Implications for secondary school educators. J Spec Educ Technol. 2003;18(4):29-44.

3. Pascarella ET, Pierson CT, Wolniak GC, Terenzini PT. First-generation college students: Additional evidence on college experiences and outcomes. J High Educ. 2004 May 1;75(3):249-284.

4. Wagner M, Newman L, Cameto R, Garza N, Levine P. After high school: A first look at the postschool experiences of youth with disabilities. A report from the national longitudinal transition study-2 (NLTS2). Menlo Park: CA: SRI International; 2005 Apr.

5. Miller-Shaul S. The characteristics of young and adult dyslexics readers on reading and reading related cognitive tasks as compared to normal readers. Dyslexia Int J Res Pract. 2005;11(2):132151.
6. Quick RL. Exploring faculty perceptions toward working with academically vulnerable college students. Coll Q. 2013;16(4):1-12.

7. Lefly DL, Pennington BF. Spelling errors and reading fluency in compensated adult dyslexics. Ann Dyslexia. 1991 Jan 1;41(1):141162.

8. Wilson AM, Lesaux NK. Persistence of phonological processing deficits in college students with dyslexia who have age-appropriate reading skills. J Learn Disabil. 2001;34(5):394-400.

9. Holmqvist K, Nyström M, Andersson R, Dewhurst R, Jarodzka H, Weijer J van de. Eye Tracking: A comprehensive guide to methods and measures. Oxford: Oxford University Press; 2011. 671 p.

10. Rayner K. Eye movement in reading and information processing: 20 years of research. Psychol Bull. 1998;124(3):372-422.

11. De Luca M, Borrelli M, Judica A, Spinelli D, Zoccolotti P. Reading words and pseudowords: An eye movement study of developmental dyslexia. Brain Lang. 2002;80(3):617-626.

12. Hyönä J, Lorch RF, Kaakinen JK. Individual differences in reading to summarize expository text: Evidence from eye fixation patterns. J Educ Psychol. 2002;94(1):44-55.

13. Desroches AS, Joanisse MF, Robertson EK. Specific phonological impairments in dyslexia revealed by eyetracking. Cognition. 2006 Jul;100(3):B32-42.

14. Hyönä J, Olson RK. Eye fixation patterns among dyslexic and normal readers: Effects of word length and word frequency. J Exp Psychol Learn Mem Cogn. 1995 Nov;21(6):1430-1440.

15. Goff DA, Pratt C, Ong B. The relations between children's reading comprehension, working memory, language skills and components of reading decoding in a normal sample. Read Writ. 2005 Dec 1;18(7-9):583-616.

16. Stanovich KE, Cunningham AE, Feeman DJ. Intelligence, cognitive skills, and early reading progress. Read Res Q. 1984;19(3):278303.

17. Vandervelden MC, Siegel LS. Phonological recoding and phoneme awareness in early literacy: A developmental approach. Read Res Q. 1995 Oct 1;30(4):854-875.

18. Swanson HL. Adults with reading disabilities: Converting a metaanalysis to practice. J Learn Disabil. 2012 Jan;45(1):17-30.

19. Leonard LB, Weismer SE, Miller CA, Francis DJ, Tomblin JB, Kail RV. Speed of processing, working memory, and language impairment in children. J Speech Lang Hear Res. 2007 Apr;50(2):408-428.

20. Shanahan MA, Pennington BF, Yerys BE, Scott A, Boada R, Willcutt EG, et al. Processing speed deficits in attention deficit/hyperactivity disorder and reading disability. J Abnorm Child Psychol. 2006 Oct 1;34(5):584-601.

21. Catts HW, Gillispie M, Leonard LB, Kail RV, Miller CA. The role of speed of processing, rapid naming, and phonological awareness in reading achievement. J Learn Disabil. 2002 Dec 11;35(6):509.

22. Wechsler D. Wechsler Adult Intelligence Scale-Third edition (WAIS-III). San Antonio, TX: Psychological Corporations; 1997.

23. Conway ARA, Cowan N, Bunting MF, Therriault DJ, Minkoff SRB. A latent variable analysis of working memory capacity, short-term memory capacity, processing speed, and general fluid intelligence. Intelligence. 2002 Mar;30(2):163-183.

24. Lezak MD, Howieson D, Loring D. Neuropsychological assessment. Oxford: Oxford University Press; 2004. 1039 p.

25. Hoyer WJ, Stawski RS, Wasylyshyn C, Verhaeghen P. Adult age and digit symbol substitution performance: A meta-analysis. Psychol 
Aging. 2004;19(1):211-214.

26. Beidas H, Khateb A, Breznitz Z. The cognitive profile of adult dyslexics and its relation to their reading abilities. Read Writ. 2013 Oct 1;26(9):1487-1515.

27. Kail RV, Hall LK. Processing speed, naming speed, and reading. Dev Psychol. 1994;30(6):949-954.

28. Waters G, Caplan D. The relationship between age, processing speed, working memory capacity, and language comprehension. Memory. 2005 May 4;13(3/4):403-413.

29. Caplan D, Waters G. The relationship between age, processing speed, working memory capacity, and language comprehension. Memory. 2003 Sep 1;13(3-4):403-413.

30. Nelson MJ, Denny EC, Brown JI. The Nelson-Denny Reading Test: Forms A \& B. Boston, MA: Houghton Mifflin Co.; 1960.

31. Swanson HL, Zheng X, Jerman O. Working memory, short-term memory, and reading disabilities a selective meta-analysis of the literature. J Learn Disabil. 2009 May 1;42(3):260-287.

32. Brosnan M, Demetre J, Hamill S, Robson K, Shepherd H, Cody G. Executive functioning in adults and children with developmental dyslexia. Neuropsychologia. 2002;40(12):2144-2155.

33. Martin J, Colé P, Leuwers C, Casalis S, Zorman M, Sprenger-Charolles L. Reading in French-speaking adults with dyslexia. Ann Dyslexia. 2010 Dec 1;60(2):238-264.

34. Thomas JG, Milner HR, Haberlandt KF. Forward and backward recall: Different response time patterns, same retrieval order. Psychol Sci. 2003 Mar 1;14(2):169-174.

35. Reynolds CR. Forward and backward memory span should not be combined for clinical analysis. Arch Clin Neuropsychol. 1997; 12(1):29-40.

36. Grant I, Adams K. Neuropsychological assessment of neuropsychiatric and neuromedical disorders. Oxford University Press, USA; 2009. 760 p.

37. Cantiani C, Lorusso ML, Guasti MT, Sabisch B, Männel C. Characterizing the morphosyntactic processing deficit and its relationship to phonology in developmental dyslexia. Neuropsychologia. 2013 Jul;51(8):1595-1607.

38. Wiseheart R, Altmann LJP, Park H, Lombardino LJ. Sentence comprehension in young adults with developmental dyslexia. Ann Dyslexia. 2009 Dec;59(2):151-167.

39. Traxler MJ, Johns CL, Long DL, Zirnstein M, Tooley KM, Jonathan E. Individual differences in eye-movements during reading: Working memory and speed-of-processing effects. J Eye Mov Res. 2012; 5(1):1-16.

40. Jones MW, Obregón M, Kelly ML, Branigan HP. Elucidating the component processes involved in dyslexic and non-dyslexic reading fluency: An eye-tracking study. Cognition. 2008;109(3):389407.

41. Thaler V, Urton K, Heine A, Hawelka S, Engl V, Jacobs AM. Different behavioral and eye movement patterns of dyslexic readers with and without attentional deficits during single word reading. Neuropsychologia. 2009 Oct;47(12):2436-2445.

42. Torgesen, Wagner R, Rashotte C. Test of Word Reading Efficiency. Austin, TX: Pro-ED; 1999.

43. Woodcock R, McGrew K, Mather N. Woodcock-Johnson III tests of cognitive abilities. Itasca, IL: Riverside Publishing Company; 2002.

44. Baayen RH, Pipenbrock R, Gulikers L. The CELEX lexical database [webcelex]. Philadelphia, PA: University of Pennsylvania, Linguistic Data Consortium; 1995.

45. Matarazzo JD, Herman DO. Base rate data for the WAIS-R: test-retest stability and VIQ-PIQ differences. J Clin Neuropsychol. 1984 Nov;6(4):351-366.

46. Youngjohn JR, Larrabee GJ, Crook TH. Test-retest reliability of computerized, everyday memory measures and traditional memory tests. Clin Neuropsychol. 1992;6(3):276-286.

47. Wechsler D. Wechsler memory scale. San Antonio, TX: Psychological Corporation; 1987.

48. Cohen-Mimran R, Sapir S. Deficits in working memory in young adults with reading disabilities. J Commun Disord. 2007;40(2): 168-183.

49. Parekh PI, Blumenthal JA, Babyak MA, LaCaille R, Rowe S, Dancel $\mathrm{L}$, et al. Gas exchange and exercise capacity affect neurocognitive performance in patients with lung disease. Psychosom Med. 2005; 67(3):425-432.

50. Just MA, Carpenter PA. Inference processes during reading: Reflections from eye fixations. In: Senders JW, Fisher DF, Monty RA, editors. Eye movements and the higher psychological functions. Hillsdale, N.J.: Lawrence Erlbaum; 1978. p. 157-174.

51. Ellsworth CP, Muir DW. Social competence and person-object differentiation: An analysis of the still-face effect. Dev Psychol. 1993 Jan;29(1):63-73.

52. Yan T, Tourangeau R. Fast times and easy questions: the effects of age, experience and question complexity on web survey response times. Appl Cogn Psychol. 2008 Jan;22(1):51-68.

53. van Silfhout G, Evers-Vermeul J, Mak WM, Sanders TJM. Connectives and layout as processing signals: How textual features affect students' processing and text representation. J Educ Psychol. 2014 Mar 17;106(4):1036-1048.

54. Goldberg JH, Kotval XP. Computer interface evaluation using eye movements: methods and constructs. Int J Ind Ergon. 1999;24(6): 631-645.

55. Renshaw JA, Finlay JE, Tyfa D, Ward RD. Designing for visual influence: an eye tracking study of the usability of graphical management information. In: Human-Computer Interaction- INTERACT03. Oxford: IOS Press; 2003.

56. Myers R. Classical and modern regression with applications. Boston, MA: Duxbury; 1990.

57. Durbin J, Watson GS. Testing for serial correlation in least squares regression, II. Biometrika. 1951;30(3/4):159-178.

58. Sisson ED. The causes of slow reading: an analysis. J Educ Psychol. 1939 Mar;30(3):206-214.

59. Underwood G, Hubbard A, Wilkinson H. Eye fixations predict reading comprehension: The relationships between reading skill, reading speed, and visual inspection. Lang Speech. 1990 Jan;33(1): 69-81.

60. Sheffield BB. The structured flexibility of Orton-Gillingham. Ann Dyslexia. 1991 Jan;41(1):41-54.

61. Mokhtari K, Reichard CA. Assessing students' metacognitive awareness of reading strategies. J Educ Psychol. 2002;94(2):249259.

62. Kirby JR, Silvestri R, Allingham BH, Parrila R, La Fave CB. Learning strategies and study approaches of postsecondary students with dyslexia. J Learn Disabil. 2008;41(1):85-96.

63. Altmann LJ, Lombardino LJ, Puranik C. Sentence production in 
students with dyslexia. Int J Lang Commun Disord. 2008;43(1):5576.

64. Georgiou GK, Parrila R, Kirby JR, Stephenson K. Rapid naming components and their relationship with phonological awareness, orthographic knowledge, speed of processing, and different reading outcomes. Sci Stud Read. 2008 Oct 22;12(4):325-350.

65. Moura O, Simões MR, Pereira M. Executive functioning in children with developmental dyslexia. Clin Neuropsychol. 2015;28(sup1): 20-41.

66. Bull R, Johnston RS. Children's arithmetical difficulties: contributions from processing speed, item identification, and short-term memory. J Exp Child Psychol. 1997 Apr;65(1):1-24.

67. Mayes SD, Calhoun SL. Learning, attention, writing, and processing speed in typical children and children with ADHD, autism, anxiety, depression, and oppositional-defiant disorder. Child Neuropsychol. 2007 Oct 29;13(6):469-493.

68. Ofiesh NS. Using processing speed tests to predict the benefit of extended test time for university students with learning disabilities. J Postsecond Educ Disabil. 2000;14(1):39-56.

69. Jong CGW de, Voorde SVD, Roeyers H, Raymaekers R, Oosterlaan J, Sergeant JA. How distinctive are ADHD and RD? Results of a double dissociation study. J Abnorm Child Psychol. 2009 Oct 1;37 (7):1007-1017.

70. Fischbach A, Könen T, Rietz CS, Hasselhorn M. What is not working in working memory of children with literacy disorders? Evidence from a three-year-longitudinal study. Read Writ. 2014 Feb 1; 27(2):267-286.

71. Kibby MY, Marks W, Morgan S, Long CJ. Specific impairment in developmental reading disabilities: A working memory approach. J Learn Disabil. 2004 Jul 1;37(4):349-363.

72. Seigneuric A, Ehrlich M-F. Contribution of working memory capacity to children's reading comprehension: A longitudinal investigation. Read Writ. 2005 Dec 1;18(7-9):617-656.

73. Arrington CN, Kulesz PA, Francis DJ, Fletcher JM, Barnes MA. The contribution of attentional control and working memory to read- ing comprehension and decoding. Sci Stud Read. 2014 Oct 9; 18(5):325-346.

74. Alloway TP, Wootan S, Deane P. Investigating working memory and sustained attention in dyslexic adults. Int J Educ Res. 2014; 67:11-7.

75. Oakhill JV, Cain K, Bryant PE. The dissociation of word reading and text comprehension: Evidence from component skills. Lang Cogn Process. 2003;18(4):443-468.

76. Denckla MB, Cutting LE. History and significance of rapid automatized naming. Ann Dyslexia. 1999 Dec;49(1):29-42.

77. Breznitz Z. Speed of phonological and orthographic processing as factors in dyslexia: Electrophysiological evidence. Genet Soc Gen Psychol Monogr. 2003 May;129(2):183-206.

78. Breznitz Z. Fluency in Reading: Synchronization of Processes. Mahwah, NJ: Lawrence Erlbaum Associates; 2006. 327 p.

79. Breznitz Z, Misra M. Speed of processing of the visual-orthographic and auditory-phonological systems in adult dyslexics: The contribution of "asynchrony" to word recognition deficits. Brain Lang. 2003 Jun;85(3):486-502.

80. Meyler A, Breznitz Z. Impaired phonological and orthographic word representations among adult dyslexic readers: Evidence from event-related potentials. J Genet Psychol. 2005 Jun 1;166(2): 215-240.

81. Engle RW. Working memory capacity as executive attention. Curr Dir Psychol Sci. 2002 Feb 1;11(1):19-23.

82. Kane MJ, Bleckley MK, Conway AR, Engle RW. A controlled-attention view of working-memory capacity. J Exp Psychol Gen. 2001; 130(2):169-183.

83. Unsworth N, Spillers GJ. Working memory capacity: Attention control, secondary memory, or both? A direct test of the dualcomponent model. J Mem Lang. 2010 May;62(4):392-406.

84. Engle RW, Kane MJ. Executive attention, working memory capacity, and a two-factor theory of cognitive control. Psychol Learn Motiv. 2004;44:145-200. 